\title{
PERFIL SENSORIAL E PREFERÊNCIA DO IOGURTE DE LEITE DE BÚFALA
}

\author{
MARTA REGINA VERRUMA-BERNARDI* \\ NÁDIA C. M. BRANCO*** \\ DANIELE M. J. MAROTE**** \\ ROSIRES DELIZA***** \\ KÁTIA GOMES DE LIMAARAÚJO*****

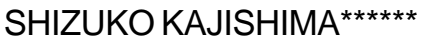

\begin{abstract}
Este estudo teve como objetivo levantar atributos sensorias e avaliar a preferência do consumidor por amostras de iogurte preparados a partir de leites de búfala, de vaca e da mistura dos dois leites. De acordo com os resultados da análise descritiva quantitativa, os três iogurtes diferiram significativamente $(p<0,05)$ entre si para os atributos cor (amarela) e aparência (espumosa). O iogurte de leite de búfala diferiu dos demais quanto ao aroma (característico de coalhada), sabor (gorduroso e amargo) e viscosidade, porém para cremosidade o iogurte integral de leite de búfala não diferiu do misto. No teste de preferência os iogurtes sem adição de sabor não apresentaram diferença significativa entre si, sendo menos preferidos que os produtos adicionados de sabor goiaba que também não diferiram estatisticamente entre si. Os resultados do Mapa Interno de Preferência dos seis iogurtes testados mostraram clara preferência dos participantes pelos iogurtes aromatizados com goiaba. Dentre esses, verificou-se tendência da preferência do iogurte de leite de búfala, seguido pelos iogurtes misto e de leite de vaca.
\end{abstract}

PALAVRAS-CHAVE: IOGURTE; LEITE DE BÚFALA; ANÁLISE SENSORIAL.

* $\quad$ Professora, Departamento de Tecnologia Agroindustrial e Sócio-Economia Rural, Universidade Federal de São Carlos (UFSCar), Araras-SP (veruma @cca.ufscar.br).

** Ex-alunas do curso de Nutrição, Universidade Federal Fluminense, (UFF), Niterói - RJ.

*** Pesquisadora da EMBRAPA Agroindústria de Alimentos, Rio de janeiro - RJ (rodeliza@ctaa.embrapa.br).

**** Professora, Faculdade de Farmácia, UFF, Niterói - RJ (klima@vm.uff.br). 


\section{INTRODUÇÃO}

O rebanho bubalino brasileiro enquadra-se entre os dos animais domésticos que apresentam maior índice de crescimento (IBGE, 2003). Dados da EMBRAPA (2004) estimam que o número de cabeças gira em torno de 3,5 milhões. SAMPAIO NETO et al. (2001) afirmaram que a taxa anual de crescimento desse rebanho no Brasil alcança $10 \%$. Segundo a EMBRAPA (2004) essa taxa de crescimento é quatro vezes maior que a do rebanho de bovinos.

Os bubalinos exibem produtividade leiteira economicamente superior aos zebuínos, ou seja, cada litro de leite é produzido com menor custo. Tal fato não se justifica apenas pela maior produção por animal e maior número de fêmeas em lactação por ano, mas pela sua rusticidade extraordinária. Os bubalinos aproveitam melhor a forragem de qualidade inferior e resistem às mais adversas condições climáticas, com marcante resistência às doenças (NASCIMENTO e CARVALHO, 1993). Entretanto, é no aproveitamento industrial do leite de búfala que reside sua grande importância. Os produtos lácteos apresentam qualidade diferenciada dos de leite bovino como, por exemplo, o queijo Mozzarella e o iogurte. Além disso, seu rendimento industrial extraordinário chega a suplantar o do leite bovino em mais de 40\% (NADER FILHO et al., 1984).

A utilização do leite de búfala na preparação de derivados tem sido pesquisada em diferentes regiões do mundo (LAXMINARAYANA e DASTUR, 1968) devido ao seu alto valor nutricional. Na sua composição são encontrados elevados níveis de sólidos totais, gordura, proteína, cálcio e fósforo que elevam os rendimentos na fabricação de queijos, produtos fermentados, leite em pó, manteiga, doce de leite e sorvete (FAO, 1991). O conhecimento dos elevados índices de produtividade e do alto valor nutritivo do leite de búfala tornaram-no valioso para o consumo in natura e para a elaboração de derivados (VERRUMA, OLIVEIRA e SALGADO, 1993).

O iogurte, importante derivado lácteo para a alimentação humana, é elaborado tradicionalmente no Brasil a partir do leite bovino. A produção de iogurte constitui excelente alternativa para o aproveitamento do leite de búfala, embora possam ocorrer problemas na sua aceitação devido ao elevado teor de gordura desse leite (CUNHA-NETO, 2003). 
Produto da fermentação láctica, o iogurte está presente na dieta humana desde tempos remotos, quando a fermentação era utilizada como forma de preservação do leite (ALAIS, 1985). Desde que Metchnikoff sugeriu no início do século, que o consumo regular de leites fermentados poderia oferecer beneficios à saúde do consumidor, as possíveis propriedades profiláticas e terepêuticas de produtos similares têm sido especuladas (TAMINE, MARSHALL e ROBINSON, 1995). De acordo com HUNGER e PEITERSEN (1992), novos produtos nessa linha tem sido desenvolvidos considerando os aspectos da saúde e boas características de aroma, sabor e textura.

ALM (1991) descreveu que os leites fermentados contém, geralmente, as mesmas quantidades de nutrientes que o leite, porém a fermentação torna alguns compostos mais disponíveis para absorção. Assim, os efeitos fisiológicos e nutricionais dos leites fermentados favorecem 0 aumento da digestibilidade e a absorção de proteínas do leite. Estudos sobre a digestibilidade in vitro de iogurte de leite de búfala mostraram valores pouco superiores $(93,50 \%)$ em relação ao elaborado com leite de vaca $(91,50 \%)$ (VERRUMA, OLIVEIRA e SALGADO, 1993).

O presente estudo teve como objetivo identificar os atributos que melhor descrevessem as características sensoriais de iogurtes preparados a partir de leite de búfala e de leite de vaca, mediante análise descritiva quantitativa. Além disso, avaliou-se a preferência do consumidor pelas amostras de iogurte adicionadas ou não de sabor goiaba.

\section{MATERIAL E MÉTODOS}

\subsection{ELABORAÇÃO DOS IOGURTES}

Foram utilizados leites integrais de búfala e de vaca, obtidos num sítio próximo a cidade de Itaguaí (RJ). Aplicou-se a metodologia descrita por GARCIA, VAN DENDER e MORENO (1986) para obter os seguintes produtos: logurte $100 \%$ de leite de búfala (ILB), iogurte $50 \%$ de leite de búfala e $50 \%$ de leite de vaca (IM) e iogurte $100 \%$ de leite de vaca (ILV). Utilizou-se fermento lácteo Rich ${ }^{\circledR}$, constituído de culturas de Lactobacillus delbrueckii ssp bulgaricus e Streptococcus thermophilus, da Empresa Christian Hansen - Valinhos (SP). 


\subsection{ANÁLISE SENSORIAL}

Para a avaliação sensorial das amostras adotou-se a Análise Descritiva Quantitativa (ADQ), adaptada da metodologia descrita por STONE e SIDEL (1985). Os testes foram realizados no horário de 9 às 13 horas no Laboratório de Alimentos e Dietética da Faculdade de Nutrição da Universidade Federal Fluminense (UFF).

\subsubsection{Desenvolvimento da terminologia descritiva}

Participaram do estudo dez julgadores, selecionados pelo interesse e disponibilidade no período de realização da análise, além de conhecimento prévio de análise sensorial. Cerca de $20 \mathrm{~g}$ de cada iogurte foram colocados em copos plásticos, codificados com números de três dígitos e servidos em temperatura de geladeira $\left(10^{\circ} \mathrm{C}\right)$ aos participantes. Serviu-se também água mineral em temperatura ambiente para lavar o palato entre uma amostra e outra. Efetuou-se o levantamento de atributos pelo método de Rede (MOSKOWITZ, 1983). As amostras foram apresentadas em três sessões e solicitado aos julgadores que anotassem as similaridades e as diferenças entre amostras, mediante ficha adequada para o levantamento de atributos.

\subsubsection{Treinamento da equipe}

Após cada julgador ter levantado os termos descritivos, a equipe reuniuse para discuti-los. Nesta etapa, os termos que expressavam o mesmo significado foram agrupados em um só atributo e retirados aqueles poucos utilizados pela equipe. No final das sessões, gerou-se a lista de termos descritivos com os respectivos extremos da escala utilizada. Durante o treinamento, os julgadores foram solicitados a avaliar a intensidade de cada atributo sensorial das amostras dos iogurtes. Para a avaliação usou-se escala não-estruturada de $9 \mathrm{~cm}$, ancorada nos extremos com termos definidos pela equipe (STONE e SIDEL, 1985).

\subsubsection{Verificação da performance dos julgadores}

Após o treinamento, os julgadores avaliaram as amostras com três repetições, utilizando a ficha desenvolvida. Os julgadores foram selecionados em função da habilidade para discriminar as amostras e repetibilidade nas avaliações. Os julgadores que apresentaram 
probabilidade de $F_{\text {amostras }}$ não-significativa $(p>0,05)$ ou $F_{\text {repeticão }}$ significativa $(p<0,05)$ em mais de um atributo foram eliminados da equipe. Neste estudo foram excluídos dois julgadores (DAMÁSIO e COSTEL, 1991).

\subsubsection{Avaliação sensorial dos iogurtes}

As amostras foram avaliadas em relação aos atributos mostrados no Quadro 1, mediante escala não-estruturada de $9 \mathrm{~cm}$, sendo apresentadas monadicamente em ordem balanceada (STONE e SIDEL, 1985). Os testes foram realizados em cabines individuais adaptadas a fim de manter o isolamento de cada julgador, sob iluminação branca. Os julgadores utilizaram água mineral para lavar o palato entre uma amostra e outra.

\subsection{TESTE DE PREFERÊNCIA}

Realizou-se o teste de preferência com três amostras de iogurte com e sem adição de sabor de goiaba (doce de goiaba em calda $30^{\circ}$ Brix), totalizando seis amostras. Quarenta e oito consumidores de produtos lácteos em geral foram convidados a participar do estudo, conduzido na Faculdade de Nutrição da UFF, na cidade de Niterói RJ. Assim, 28 mulheres e 20 homens com idade entre 18 e 50 anos avaliaram os iogurtes. Cerca de $20 \mathrm{~g}$ de cada iogurte foram colocados em copos plásticos, codificados com números de três dígitos e servidos em temperatura de geladeira $\left(10^{\circ} \mathrm{C}\right)$ aos $p$ articipantes. Também serviuse água mineral em temperatura ambiente para lavar o palato entre uma amostra e outra. Segui-se a ordem balanceada de apresentação das amostras e delineamento de blocos completos, segundo MAcFiE et al. (1989). As amostras foram apresentadas monadicamente e o teste realizado entre $14 \mathrm{~h}$ e $17 \mathrm{~h}$. Utilizou-se a escala hedônica de sete pontos, variando de "gostei muito" a "desgostei muito".

\subsection{ANÁLISE ESTATÍSTICA}

Os dados da ADQ foram submetidos a análises de variância, sendo aplicado o teste de Tukey para checar diferenças entre as médias (SAS, 1989). Os dados da preferência foram analisados mediante ferramenta denominada Mapa Interno da Preferência do XLSTAT-MX do Excel. Com essa ferramenta foi realizada a análise de componentes principais (ACP) da matriz de covariância de consumidores por 
produto, agrupando os participantes conforme preferência similar (GREENHOFF e MacFIE, 1994). Desse modo, os dados individuais dos participantes foram incorporados na análise dos resultados, não se referindo (portanto) às médias dos produtos.

\section{QUADRO 1 - ATRIBUTOS E DEFINIÇÕES UTILIZADOS}

\begin{tabular}{|c|c|}
\hline ATRIBUTOS & DEFINIÇÃO \\
\hline \multicolumn{2}{|l|}{ APARÊNCIA } \\
\hline Cor amarela & $\begin{array}{l}\text { Refere-se à intensidade da cor amarela percebida nos produtos } \\
\text { Referência: creme de leite }\end{array}$ \\
\hline Aparência espumosa & Refere-se a aparência de leite enriquecido batido \\
\hline Aparência homogênea & Refere-se a aparência de requeijão cremoso \\
\hline \multicolumn{2}{|l|}{ AROMA } \\
\hline Característico & Refere-se ao aroma característico de iogurte natural de leite de vaca \\
\hline Ácido & Refere-se ao grau de acidez do iogurte, \\
\hline Coalhada & Refere-se ao aroma característico de coalhada \\
\hline \multicolumn{2}{|l|}{ SABOR } \\
\hline Gorduroso & $\begin{array}{l}\text { Refere-se ao sabor de gordura láctea, que permanece por um tempo } \\
\text { na boca }\end{array}$ \\
\hline $\begin{array}{l}\text { Característico de } \\
\text { iogurte de leite de vaca }\end{array}$ & $\begin{array}{l}\text { Refere-se ao sabor característico de iogurte natural elaborado com } \\
\text { leite de vaca }\end{array}$ \\
\hline Ácido & $\begin{array}{l}\text { Refere-se ao gosto ácido percebido no instante que a amostra entra } \\
\text { em contato com as papilas gustativas, característico do ácido lático }\end{array}$ \\
\hline Sabor residual lácteo & $\begin{array}{l}\text { Refere-se ao sabor lácteo que permanece por um tempo após } \\
\text { degustação }\end{array}$ \\
\hline Amargo & Refere-se ao gosto amargo percebido na degustação \\
\hline \multicolumn{2}{|l|}{ TEXTURA } \\
\hline $\begin{array}{l}\text { Cremosidade } \\
\text { Viscosidade }\end{array}$ & $\begin{array}{l}\text { Refere-se a textura cremosa } \\
\text { Fraca: iogurte natural } \\
\text { Forte: especialidade láctea } \\
\text { Refere-se a textura viscosa grossa peqaiosa do iogurte }\end{array}$ \\
\hline
\end{tabular}

\section{RESULTADOS E DISCUSSÃO}

\subsection{ANÁLISE SENSORIAL DESCRITIVA}

As médias dos 12 atributos sensoriais elicidados pelos julgadores 
para descrever os iogurtes estão apresentados no Quadro 2.

Os três iogurtes diferiram significativamente $(p<0,05)$ entre si para os atributos cor amarela e aparência espumosa. O ILV apresentou cor amarela mais intensa, o ILB cor branca e o IM cor intermediária. Quanto à aparência espumosa, o ILV apresentou-se mais espumoso, seguido pelo IM. Em relação aos atributos aparência homogênea, aroma ácido e gosto ácido não houve diferença significativa entre os iogurtes avaliados.

\section{QUADRO 2 - MÉDIAS DOS ATRIBUTOS SENSORIAIS DESCRITOS PARA OS IOGURTES}

\begin{tabular}{|ccccc|}
\hline & Atributos & IL B & IM & ILV \\
\hline Aparência & Coramarela & $0,8 \mathrm{c}$ & $4,5 \mathrm{~b}$ & $7,6 \mathrm{a}$ \\
& Espumosa & $1,6 \mathrm{c}$ & $4,5 \mathrm{~b}$ & $6,6 \mathrm{a}$ \\
& Homogênea & $6,5 \mathrm{a}$ & $6,1 \mathrm{a}$ & $5,8 \mathrm{a}$ \\
\hline \multirow{2}{*}{ Aroma } & Característico & $5,6 \mathrm{~b}$ & $7,3 \mathrm{a}$ & $8,3 \mathrm{a}$ \\
& Coalhada & $3,6 \mathrm{~b}$ & $5,6 \mathrm{a}$ & $7,0 \mathrm{a}$ \\
& Ácido & $5,4 \mathrm{a}$ & $5,7 \mathrm{a}$ & $6,2 \mathrm{a}$ \\
\hline \multirow{2}{*}{ Sabor } & Gorduroso & $7,4 \mathrm{a}$ & $3,9 \mathrm{~b}$ & $3,8 \mathrm{~b}$ \\
& Característico & $4,8 \mathrm{~b}$ & $6,3 \mathrm{ab}$ & $7,0 \mathrm{a}$ \\
& Ácido & $5,2 \mathrm{a}$ & $5,4 \mathrm{a}$ & $6,9 \mathrm{a}$ \\
& Residuallácteo & $5,1 \mathrm{~b}$ & $4,6 \mathrm{ab}$ & $6,9 \mathrm{a}$ \\
& Amargo & $8,0 \mathrm{a}$ & $3,9 \mathrm{~b}$ & $3,0 \mathrm{~b}$ \\
\hline Textura & Cremosidade & $5,6 \mathrm{~b}$ & $4,8 \mathrm{~b}$ & $8,0 \mathrm{a}$ \\
& Viscosidade & $8,5 \mathrm{a}$ & $4,3 \mathrm{~b}$ & $5,2 \mathrm{~b}$ \\
\hline
\end{tabular}

Médias na mesma linha seguidas por letras iguais não diferem significativamente $(p<0,05)$.

ILB = iogurte de leite búfala; ILV = iogurte leite vaca; IM = iogurte misto $(50 \%$ leite de vaca e $50 \%$ leite búfala).

Para os atributos aroma característico, lácteo e coalhada, verificouse diferença significativa apenas entre o ILV e o IM em relação ao ILB. O ILV e o IM apresentaram aroma característico, lácteo e de coalhada mais fortes. 
Quanto ao sabor gorduroso e amargo houve diferença significativa entre o ILV e o IM comparado com o ILB. Os dois primeiros (ILV e IM) apresentaram sabor gorduroso e amargo mais fracos. Para o sabor característico e o residual lácteo houve diferença significativa entre o ILB e o ILV, tendo o último sido considerado pela equipe de julgadores como mais forte nos atributos mencionados.

Observou-se diferença significativa para cremosidade entre os produtos preparados com leite de búfala e misto em relação ao iogurte processado com leite de vaca. O ILV salientou-se como o mais cremoso. Para a viscosidade, o ILB foi considerado o mais viscoso, diferindo estatisticamente dos iogurtes de leite de vaca e misto. GANGULI (1979) e VERRUMA, OLIVEIRA e SALGADO (1993) relataram que o iogurte de leite de búfala apresenta maior viscosidade, provavelmente, decorrente da maior quantidade de sólidos totais do leite de búfala (cerca de $18 \%$ ) em relação ao leite de vaca $( \pm 13 \%)$. Desta maneira, na elaboração do iogurte com leite de búfala não é necessária a adição de leite em pó, procedimento normalmente utilizado no processamento de iogurte a partir de leite de vaca para melhorar sua consistência.

O alto teor de caseína do leite de búfala, que explica a elevada consistência (semelhante a uma "papa"), nem sempre agrada ao consumidor, principalmente se o produto for envasado em garrafas plásticas. Os atributos sensoriais definem maior ou menor aceitação do produto pelo consumidor (SHEPHERD, 2001) e PENNA et al. (1997) consideram a viscosidade como fator determinante na aceitação de iogurte.

Para FUCHS et al. (2005), a firmeza do iogurte constitui atributo importante na aceitação do produto pelo consumidor. De acordo com O'NEIL, KLEYN e HARE (1979) e PENNA et al. (1997), o iogurte deve apresentar textura suave e corpo viscoso sem fissuras, além de firmeza e coesão para ser consumido com colher.

\subsection{TESTE DE PREFERÊNCIA}

A média das notas da preferência para os seis iogurtes avaliados são mostradas no Quadro 3. 
No Mapa Interno da Preferência dos seis iogurtes estudados (Figura 1) as duas primeiras dimensões responderam por $85,3 \%$ da variância total (dimensão 1:73,7\% e dimensão 2:11,6\%). Os resultados mostraram clara preferência dos participantes pelos iogurtes aromatizados com goiaba. Dentre esses, o iogurte de leite de búfala obteve a preferência dos indivíduos localizados no quadrante superior direito, já os iogurtes mistos e de vaca foram preferidos pelos indivíduos situados no quadrante inferior direito.

QUADRO 3 - MÉDIA E ERRO-PADRÃO DAS NOTAS PARA A PREFERÊNCIA DOS SEIS IOGURTES ESTUDADOS

\begin{tabular}{|lcc|}
\hline Produto & Preferência & \\
\hline & Média & Erro-padrão \\
\hline A (100\% leite de búfala + goiaba) & $4,6^{\mathrm{a}}$ & 0,31 \\
B (misto + goiaba) & $4,6^{\mathrm{a}}$ & 0,28 \\
C (100\% leite de vaca + goiaba) & $5,1^{\mathrm{a}}$ & 0,27 \\
D (leite de búfala) & $1,6^{\mathrm{b}}$ & 0,16 \\
E (misto) & $1,3^{\mathrm{b}}$ & 0,09 \\
F (leite de vaca) & $1,6^{\mathrm{b}}$ & 0,12 \\
\hline
\end{tabular}

Existem poucos trabalhos sobre a avaliação sensorial de iogurte de leite de búfala e alguns evidenciam menor preferência pelo seu sabor em relação ao iogurte elaborado com leite de vaca. CUNHA-NETO (2003) observou que o iogurte de leite de búfala elaborado com $3 \%$ de gordura obteve bom desempenho sensorial. Estudos descritos por YABU et al. (1988) mostraram participação decisiva do sabor na aceitação de iogurtes processados a partir de leite de búfala. Porém, produto resultante da mistura de 50:50\% de leite de búfala e de vaca não teve seu desempenho comprometido pelo sabor e pela consistência. 


\section{FIGURA 1 - MAPA INTERNO DA PREFERÊNCIA DOS IOGURTES ESTUDADOS}
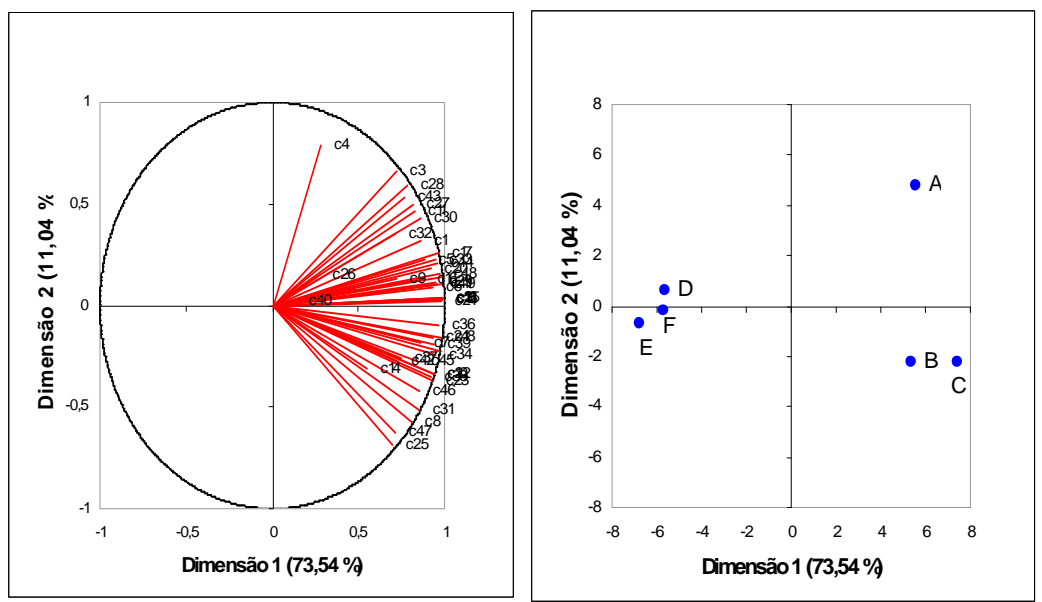

$(A=100 \%$ LEITE DE BÚFALA + GOIABA $\mathrm{B}=$ MISTO + GOIABA $; \mathrm{C}=100 \%$ LEITE DE VACA + GOIABA; D = 100\% LEITE DE BÚFALA; E = MISTO; F = 100\% LEITE DE VACA).

\section{CONCLUSÃO}

De acordo com os resultados conclui-se que os três iogurtes analisados diferiram para os atributos cor (amarela) e aparência (espumosa), e o iogurte de leite de búfala diferiu dos demais quanto ao aroma característico de coalhada, sabor (gorduroso e amargo) e viscosidade. Para a cremosidade o iogurte integral de leite de búfala não diferiu do misto, mostrando que a composição física e química afeta as características sensoriais e diferencia os iogurtes estudados.

Os resultados do Mapa Interno da Preferência dos seis iogurtes estudados mostraram clara preferência dos participantes pelos iogurtes aromatizados com goiaba. Dentre esses, verificou-se tendência de preferência pelo iogurte de leite de búfala, seguido pelos iogurtes misto e de leite de vaca. 


\section{Abstract \\ SENSORIAL PROFILE AND PREFERENCE OF YOGURT MADE WITH BUFFALO MILK}

This work had as objective to raise sensory attributes and to evaluate the consumer preference of yogurt samples made from buffalo milk, cow milk and mixture of the two milks. The results of quantitative descriptive analysis showed that the samples were significantly different $(p<0,05)$ for attributes such as color (yellow) and appearance (foamy). The buffalo milk yogurt was different from the others in relation to the aroma (characteristic of curd), taste (fatty and bitter) and viscosity, but there was no difference for creaming of yogurts made from buffalo milk and mixture. In the preference test, the yogurts without addition of taste did not show significant difference between them, and they were less preferred than the ones added of guava taste, that also did not show difference between them. The results of Preference Internal Chart for the six studied yogurts showed clear consumers preference by yogurts added of guava taste. Among those, a preference tendency was observed for buffalo milk yogurt, followed by the mixture of buffalo/cow milks and cow milk.

KEY-WORDS: YOGURT; BUFFALO MILK; SENSORIAL ANALYSIS.

\section{REFERÊNCIAS}

1 ALAIS, C.H. Ciência de la leche: princípios de técnica lechera. Barcelona: Revertè, 1985.

2 ALM, L. The therapeutic effects of various cultures: an overview. In: ROBINSON, R.K. Therapeutic properties of fermented milks. London: Elsevier, 1991. p.45-64.

3 CUNHA-NETO, O.C. Avaliação do iogurte natural produzido com leite de búfala contendo diferentes níveis de gordura. Pirassununga, 2003. 58 p. Dissertação (Mestrado), Faculdade de Zootecnia e Engenharia de Alimentos, Universidade de São Paulo.

4 DAMÁSIO, M.H.; COSTEL, E. Análises sensorial descriptivo generación de descriptores y selección de catadores. Revista Agroquímica y Tecnologia de Alimentos, v.31, n.2, p.165-178, 1991. 
5 EMBRAPA. Amazônia Oriental. Búfalo: bubalus bubalis. Disponível em: www.cpatu.embrapa.br/bufalo/bufalo.htm. Acesso em: 05 jun. 2004.

6 FAO. Food and Agriculture Organization. 0 búfalo. Brasília: Ministério da Agricultura; São Paulo: Associação Brasileira dos Criadores de Búfalos. 1991. 320 p. (FAO. Produção animal e saúde).

7 FUCHS, R.H.B.; BORSATO, D.; B.E.; HAULY, M.C.O. "logurte" de soja suplementado com oligofrutose e inulina. Revista de Ciência e Tecnologia de Alimentos, v.25, n.1, 2005. Disponível em: http://www.scielo.br/pdf/cta/v25n1/a28v25n1.pdf. Acesso em: 05 jun. 2005.

8 GANGULI, U.C. Tecnologia do leite de búfala. Revista dos Criadores, v.65, n.5, p.42-50, 1981.

9 GARCIA, S.; Van DENDER, A.G.F.; MORENO, I. Tecnología de laticínio a nível de fazenda. In: RUSSO, H.G. Bubalinocultura. Campinas: CATI, 1986. p.48-60.

10 GREENHOFF, K.; MacFIE, H.J.H. Preference mapping in practice. In: MacFIE, H.J.H.; THOMSON, D.M.H. (Ed.). Measurement of food preferences. London: Blackie Academic and Professional, 1994. p.137- 165 .

11 HUNGER, W.; PEITERSEN, N. New technical aspects of preparation of starter cultures. Bulletin International Dairy Federation, n.277, p. 4-16, 1992.

12 IBGE. Instituto Brasileiro de Geografia e estatística Censo Agropecuário de 1996: quantidade de animais. Disponível em: www.sidra.ibge.gov.br/bda/ pecua/default.asp?z=t\&o=12. Acesso em :13 jun. 2004.

13 LAXMINARAYANA, H; DASTUR, N.N. Buffaloes milk and milk products I. Dairy Science Abstracts, v.30, n.4, p.177-186, 1968.

14 MacFIE, H. J. H. et al. Designs to balance the effect of order of 
presentation and first-order carry-over effects in hall tests. Journal of Sensory Studies, n.4, p129-148. 1989.

15 MOSKOWITZ, H.R. Product testing and sensory evalution of foods. Westport: Food \& Nutrition Press, 1983. 605 p.

16 NADER FILHO, A.; SCHOCKEN-ITURRINO, R.P.; ROSSI JUNIOR, O.D.; CAVAGLIANO, C.P.G. Estudo da variação do ponto crioscópico do leite de búfala. Revista do Instituto de Laticínios Cândido Tostes, v.39, n. 234, p.37-39, 1984.

17 NASCIMENTO, C.; CARVALHO, L.O.M. Criação de búfalos: alimentação, manejo, melhoramento e instalações. Brasília, D.F: MARA/EMBRAPA, 1993.

18 O'NEIL, J.M.; KLEYN, D.H.; HARE, L.B. Consistency and compositional characteristics of commercial yoghurts. Journal Dairy Science, v. 62, p.1032-1036, 1979.

19 PENNA, A.L.B.; OLIVEIRA, M.N.; BARUFFALDI, R. Análise de consistência de iogurte: correlação entre medida sensorial e instrumental. Ciência e Tecnologia de Alimentos, v.17, n.2, p.98-101, 1997.

20 SAMPAIO NETO, J.C.; MARTINS FILHO, R.; LÔBO, R.N.B.; TONHATI, H. Avaliação dos desempenhos produtivos e reprodutivos de um rebanho bubalino no Estado do Ceará. Revista Brasileira de Zootecnia, v.30, n.2, p.368-373, 2001.

21 SHEPHERD, R. Does taste determine consumption? Understanding the psychology of food choice. In: FREWER, L. J.; RISVIK, E.; SCHIFFERSTEIN, H. (Eds.). Food people and society: a european perspective of consumers' food choice. Berlin: Springer, 2001. p.117-130.

22 STONE, H.S.; SIDEL, J.L. Sensory evalution practices. London: Academic Press, 1985. 311 p.

23 SAS INSTITUTE INC. SAS/STAT: user's guide. Cary, 1989. 846 p. Version 6. 
24 YABU; M.C.; SCHOLZ, M.B.S.; RAPACI, M.; ANTUNES, L.A.F. Características físico-químicas e sensoriais de iogurte produzido de misturas de leite bovino e bubalino. Revista do Instituto Laticínios Cândido Tostes, v.43, n.258, p.33-34, 1988.

25 TAMINE, A.Y.; MARSHALL, V.M.E.; ROBINSON, R.K. Microbiological and aspects of milks fermented by bifidobacteria. Journal of Dairy Research, n.62, p.151-187, 1995.

26 VERRUMA, M.R.; OLIVEIRA, A.J; SALGADO, J.M. Avaliação química e nutricional do queijo mozzarella e iogurte de leite de búfala. Scientia Agricola, v.50, n.3, p.438-443, 1993. 